\title{
Cancer-Associated Adipocytes Release FUCA2 to Promote Aggressive Phenotype in Triple-Negative Breast Cancer
}

\section{Qun Liu}

China Medical University

Hui-ting Dong

China Medical University

\section{Tingting Zhao}

China Medical University

\section{Fan Yao}

China Medical University

\section{Yingying Xu}

China Medical University

\section{Bo Chen}

China Medical University

\section{Yunfei Wu}

China Medical University

Feng Jin

China Medical University

Peng Xing ( $\nabla$ pxing@cmu.edu.cn )

China Medical University

\section{Research article}

Keywords: adipocyte, FUCA2, TM9SF3, secretion, triple-negative breast cancer

Posted Date: June 18th, 2021

DOl: https://doi.org/10.21203/rs.3.rs-617083/v1

License: (1) (i) This work is licensed under a Creative Commons Attribution 4.0 International License. Read Full License 


\section{Abstract}

Background: Cancer-associated adipocytes (CAAs) have been suggested to promote tumor progression. Yet, the role of CAAs in triple-negative breast cancer (TNBC) is poorly investigated.

Methods: We compared the expression of secretory protein-encoding genes in CAAs and control adipocytes. The effect of key secretory protein(s) on TNBC cell behaviors was explored.

Results: CAAs expressed and secreted FUCA2 at greater levels than control adipocytes. When FUCA2 activity was blocked with a neutralizing antibody, TNBC cell proliferation and migration induced by CAA conditioned medium was impaired. In contrast, supplement of exogenous FUCA2 protein reinforced the proliferation, colony formation, and migration of TNBC cells. In vivo studies confirmed that FUCA2 exposure enhanced tumorigenesis and metastasis of TNBC cells. Mechanistic investigation revealed that FUCA2 induced TNBC aggressiveness through TM9SF3-dependent signaling. Depletion of TM9SF3 blocked CAA- and FUCA2-induced TNBC cell proliferation and migration. Compared to adjacent breast tissues, TNBC tissues had increased expression of TM9SF3. Moreover, high TM9SF3 expression was associated with advanced TNM stage, lymph node metastasis, and shorter overall survival of TNBC patients.

Conclusions: Altogether, CAAs secrete FUCA2 to promote TNBC growth and metastasis through interaction with TM9SF3. Inhibition of TM9SF3 may represent a potential therapeutic strategy in the treatment of TNBC.

\section{Introduction}

Triple-negative breast cancer (TNBC) lacks expression of estrogen receptor, progesterone receptor, and human epidermal growth factor receptor 2, and represents the most aggressive subtype of breast cancer $[1,2]$. The tumor microenvironment plays a pivotal role in orchestrating the malignant properties of TNBC cells $[3,4]$. Adipose tissue in the tumor microenvironment is attracting more attention because of its ability to secrete a lot of bioactive factors [5, 6]. Adipose stem cells can produce leptin to enhance invasion and metastasis of TNBC cells [7]. Cancer-associated adipocytes (CAAs) accelerate breast cancer cell invasion by producing IL-6 [8]. Despite these advances, most of the secreted factors from CAAs are poorly characterized in TNBC progression.

Alpha-L-fucosidase 2 (FUCA2) is a member of the glycosyl hydrolase 29 (GH29) family and has a fucosidase activity [9]. The enzyme is responsible for the removal of the alpha-1,6-fucose linked to $\mathrm{N}$ acetylglucosamine residue of glycoproteins. Serum FUCA2 is a potential biomarker used for early detection of esophageal squamous cell carcinoma (ESCC) and hepatocellular carcinoma (HCC) $[10,11]$. FUCA2 can be secreted by Helicobacter pylori (H. pylori)-infected gastric cancer cells and play an essential role in $H$. pylori infection [12]. However, the role of FUCA2 in breast cancer remains unclear. 
The transmembrane 9 superfamily (TM9SF) contains at least 4 members, i.e., TM9SF1-4, which are characterized by a large $\mathrm{N}$-terminal extracellular domain and 9 putative transmembrane domains [13]. Several lines of evidence have established a link between TM9SF proteins and tumor progression [1416]. TM9SF4 has been reported to alter cellular $\mathrm{pH}$ in colon cancer cells, consequently regulating drug resistance and invasiveness [14]. In breast cancer, TM9SF4 contributes to chemoresistance [15]. TM9SF3 is involved in the proliferation and invasion of human T-cell leukemia [17]. These studies suggest TM9SF proteins as a potential target for anticancer therapy.

In the current study, we identify FUCA2 as a secretory protein mediating the crosstalk between CAAs and TNBC cells. We explored the function of FUCA2 in modulating TNBC cell proliferation, colony formation, and invasion. Moreover, TM9SF3 was found to be involved in the oncogenic effects of FUCA2 on TNBC cells.

\section{Materials And Methods}

\section{Isolation of adipocytes from breast adipose tissue}

Breast adipose tissue specimens were obtained from female subjects undergoing reduction mammoplasty ( 5 cases) or tumorectomy (10 cases). An informed consent form was signed by each participant.

Adipocyte isolation was performed as described previously $[8,18]$. In brief, adipose tissue samples were minced and digested in DMEM/F12 medium containing collagenase type I (Sigma-Aldrich, St. Louis, MA, USA) at $37^{\circ} \mathrm{C}$ for $2 \mathrm{~h}$. Mature adipocytes were harvested by centrifugation at $200 \mathrm{~g}$. Adipocytes were maintained in DMEM/F12 medium supplemented with $10 \%$ fetal bovine serum (FBS; Life Technologies, Carlsbad, CA, USA). Adipocytes isolated from breast tissues adjacent to tumors were defined as CAAs, while those from the subjects without cancers were used as control adipocytes.

\section{Preparation of adipocyte-conditioned medium}

Adipocytes were cultured in DMEM/F12 medium without serum for $48 \mathrm{~h}$. The conditioned medium was centrifuged at $3000 \mathrm{~g}$ for $10 \mathrm{~min}$ to remove cell debris. Adipocyte-conditioned medium were diluted at a 1:1 ratio with DMEM/F12 medium and supplemented with 10\% FBS before use. In some experiments, anti-FUCA2 neutralizing antibody or IgG isotype control $(0.1 \mu \mathrm{g} / \mathrm{mL}$; Abcam, Cambridge, MA, USA) was added to adipocyte-conditioned medium.

\section{Brest cancer cell culture and treatment}

TNBC cell lines MDA-MB-231 and MDA-MB-468 were obtained from the Type Culture Collection of Chinese Academy of Science (Shanghai, China) and cultured in Dulbecco's modified Eagle's medium (DMEM) supplemented with 10\% FBS. For FUCA2 treatment, breast cancer cells were exposed to 100 and $200 \mathrm{ng} / \mathrm{mL}$ recombinant human FUCA2 protein or PBS (as vehicle control) and tested for proliferation, colony formation, and migration. 


\section{Quantitative PCR array assay}

We utilized a quantitative PCR array that can simultaneously profile 84 secretory protein-encoding genes. This assay was performed as described in previous studies [19]. Briefly, CAAs and control adipocytes were lysed in TRIzol reagent (Life Technologies) and extracted for total RNA, as per the manufacturer's instructions. cDNA synthesis was completed using the RevertAid First Strand cDNA Synthesis Kit (Thermo Scientific, Waltham, MA, USA). The quantitative real-time PCR array was performed using SYBR Green PCR Master Mix (Applied Biosystems, Foster City, CA, USA). Relative gene expression levels were calculated using the $2^{-\Delta \Delta C t}$ method.

\section{Quantitative PCR analysis}

RNA extraction and reverse transcription were conducted as described above. Quantitative real-time PCR analysis was performed with the following PCR primers: FUCA2 sense: 5'-GGCATTATGGTAGAACTGCCA3', FUCA2 antisense: 5'-GGGAAAGGGCTGAACTGCCAA-3'; GRN sense: 5'-TCTGTAGTCTGAGCGCTACCC-3', GRN antisense: 5'-GTTAAGGCCACCCAGCTCAC-3'; CTGF sense: 5'-CTCCTGCAGGCTAGAGAAGC-3', CTGF antisense: 5'-GATGCACTTTTTGCCCTTCTT-3'; GPX3 sense: 5'-GAGCTTGCACCATTCGGTCT-3', GPX3 antisense: 5'-GGGTAGGAAGGATCTCTGAGTTC-3'; TM9SF3 sense: 5'-GCAGTAGGCATTACTACAGTAC-3', TM9SF3 antisense: 5'-CACTAAGTGCCAGGAAGATTCC-3'; GAPDH sense: 5'-TCTCCTGCGACTTCAACAGC3', GAPDH antisense: 5'-AGTTGGGATAGGGCCTCTCTT-3'. The relative gene expression was determined after normalization toward GAPDH.

\section{Western blot analysis}

Cells extracts were prepared in ice-cold RIPA lysis buffer containing a protease and phosphatase inhibitor mixture. The lysates were centrifuged at $14,000 \mathrm{~g}$ for $30 \mathrm{~min}$ at $4{ }^{\circ} \mathrm{C}$. Protein samples were resolved by SDS-polyacrylamide gel electrophoresis. Proteins were transferred to nitrocellulose membranes and incubated with the primary antibody recognizing FUCA2 (1:500 dilution; Abcam) or GAPDH (1:2000 dilution). IRDye 800CW-conjugated goat anti-rabbit IgG was used as secondary antibody. Band signals were detected using an Odyssey infrared scanner (LI-COR Biosciences, Lincoln, NE, USA).

\section{Quantification of FUCA2 levels in conditioned media}

Adipocyte conditioned media were collected as described above. The concentrations of FUCA2 in the conditioned media were assayed using a commercially available ELISA kit. Optical density was recorded at $450 \mathrm{~nm}$.

\section{Cell proliferation assay}

MDA-MB-231 and MDA-MB-468 cells were plated at a density of $1 \times 10^{4}$ cells per well and cultured in indicated conditions for 3 days. The number of viable cells was determined every day. Cell proliferation curves were then plotted. 


\section{Colony formation assay}

TNBC cells were plated at a low density of 600 cell per well and cultured with adipocyte conditioned media for 14 days. The colonies were stained and manually counted.

\section{Cell migration assay}

TNBC cells were plated onto 12-well plates and grew to confluence. The cell monolayer was scratched with a pipette tip. The cells were cultured in indicated conditions for $24 \mathrm{~h}$. Cell proliferation was inhibited by mitomycin C (1 $\mu \mathrm{g} / \mathrm{mL}$; Sigma-Aldrich). Cells were photographed, and the percentage of wound closure was calculated from three independent experiments.

\section{Animal studies}

For tumorigenetic studies, MDA-MB-231 and MDA-MB-468 cells were treated with recombinant FUCA2 protein $(200 \mathrm{ng} / \mathrm{mL})$ or PBS for 1 week and then subcutaneously injected into nude mice. Tumor volume was measured at indicated timepoints. Tumor growth curves were plotted. Mice were sacrificed 22 days after cell injection, and tumors were collected and weighed. Luciferase-labeled MDA-MB-231 cells were used to establish a lung metastasis model. In detail, cells were treated with FUCA2 or PBS for 1 week and injected through the tail vein. Six weeks post injection, D-luciferin was intraperitoneally injected and subjected to bioluminescence imaging.

\section{siRNA screening}

Screening was performed using a custom siRNA library that targets 370 genes encoding plasma membrane proteins. Each gene was silenced with a pool of 3 independent siRNAs. In brief, transfection reagents were added to form a complex with siRNA duplexes that were pre-dispensed in plate wells. MDAMB-231 and MDA-MB-468 cells were then added to the wells (2,000 cells per well). Twenty-four hours later, transfected cells were incubated with FUCA2 $(200 \mathrm{ng} / \mathrm{mL})$ or PBS for 3 additional days. Cell viability was measured using the CellTiter-Glo Luminescent Cell Viability Assay (Promega, Madison, WI, USA). Raw data were normalized using the standard z-score method [20]. Hits were designated as having zscores of $<-2.0$.

For knockdown experiments, 2 independent siRNAs targeting FUCA2 were individually transfected to TNBC cells using Lipofectamine 3000 transfection reagent (Thermo Fisher Scientific, Rockford, IL, USA). The transfected cells were then subjected to cell proliferation, colony formation, and migration assays.

\section{Tissue specimens}

For analysis of TM9SF3 expression and clinical significance, we collected 67 paired tumor and adjacent noncancerous tissues from TNBC patients undergoing tumor resection. None of the patients received any anticancer therapy prior to surgery. 
Formalin-fixed and paraffın-embedded tissues were sectioned and deparaffinized using xylene. Antigen retrieval was performed using citrate buffer ( $\mathrm{pH}$ 6.0). Sections were incubated with anti-TM9SF3 antibody (Abcam; 1:100 dilution) at $4^{\circ} \mathrm{C}$ overnight. The sections were stained with a streptavidin-peroxidase staining kit (Zhongshan Jinqiao Company, Beijing, China). Immunoreaction was revealed by 3,3'diaminobenzidine solution. The staining results were assessed in a blind manner by 2 experienced pathologists. The staining intensity was scored as 0 (no staining), 1 (weak staining), 2 (moderate staining), and 3 (strong staining). The area of positive staining was graded as $0(<10 \%), 1(10-25 \%), 2$ $(26-50 \%)$ and $3(>50 \%)$. The final immunohistochemistry score was determined by multiplying staining intensity by percentages of positive cells. The expression of TM9SF3 were categorized as low expression (immunohistochemistry score 0-3) and high expression (immunohistochemistry score 4-9).

\section{Statistical analysis}

The results are expressed as mean \pm standard deviation of at least three independent experiments. Statistical differences were analyzed using the Student's $t$-test or ANOVA with the Bonferroni post hoc test. The relationship between TM9SF3 expression and clinicopathological characteristics was analyzed using the chi-square test. Survival analysis was performed using a Kaplan-Meier plot and Log-rank test. $P$ values below 0.05 were considered statistically significant.

\section{Results}

\section{FUCA2 expression is enhanced in CAAs from TNBC}

Given that adipocytes can produce a large number of secretory proteins [21], here we performed quantitative PCR array assays between control adipocytes and CAAs to identify differentially expressed genes that encode secretory proteins. Among the 84 genes analyzed, GRN, CTGF, and FUCA2 were upregulated and GPX3 was downregulated in CAAs (Figs. 1A and 1B). FUCA2 was selected for further studies because it shows the highest fold-induction and also, is less characterized in cancer progression. Western blot analysis confirmed the upregulation of FUCA2 at the protein level in CAAs (Fig. 1C). Most interestingly, ELISA demonstrated that the concentration of FUCA2 was significantly greater in conditioned media from CAAs than from control adipocytes (Fig. 1D). These results indicate that CAAs from TNBC are able to release abundant FUCA2 to the extracellular environment.

\section{Blocking Fuca2 Attenuates Caa-induced Aggressiveness In Tnbc Cells}

Next, we tested whether FUCA2 can mediate CAA-induced TNBC aggressive phenotype. When MDA-MB231 and MDA-MB-468 TNBC cells were exposed to CAA conditioned media, cell proliferation and colony formation were enhanced relative to exposure to control media (Figs. 2A \& 2B). Moreover, the migratory capacity of MDA-MB-231 and MDA-MB-468 cells was elevated upon stimulation with CAA conditioned 
media (Fig. 2C). Strikingly, the effects of CAA conditioned medium on TNBC cells were attenuated when an FUCA2 neutralizing antibody was added (Fig. 2). These results suggest that FUCA2 plays an essential role in mediating the tumor-promoting activity of CAAs in TNBC.

\section{Exogenous Fuca2 Drives Tnbc Cell Growth And Migration}

Next, we interrogated the effect of exogenous FUCA2 on the aggressive phenotype of TNBC cells. Our data demonstrated that the addition of recombinant FUCA2 protein to culture media led to a significant increase in the proliferation, colony formation, and migration of TNBC cells (Fig. 3). Moreover, such effect showed concentration dependence. These findings confirm the oncogenic role for FUCA2 in TNBC.

FUCA2 exposure promotes tumorigenesis and metastasis of TNBC cells in vivo

Next, we investigated the effect of FUCA2 exposure on TNBC tumorigenesis and metastasis in vivo. TNBC cells were exposed to recombinant FUCA2 protein for 1 week and then injected subcutaneously into nude mice. FUCA2-exposed TNBC cells yielded significantly larger tumors than control cells (Figs. 4A-4C). For establishment of experimental lung metastasis, luciferase-labeled MDA-MB-231 (MDA-MB-231-luc) cells were injected through the tail vein before treatment with FUCA2 protein. We found that the FUCA2exposed group showed higher bioluminescence levels in the lung than the control group (Figs. 4D and 4E)

\section{Fuca2-mediated Tnbc Aggressiveness Depends On Tm9sf3 Expression}

To determine the genes that are indispensable for FUCA2-elicited aggressive phenotype, we performed high-throughput siRNA screen in both MDA-MB-231 and MDA-MB-468 cells to target 370 genes encoding plasma membrane proteins. The screen led to an identification of 26 genes in MDA-MB-231 and 18 genes in MDA-MB-468 whose knockdown restrained FUCA2-induced cell proliferation (Fig. 5A). There were 3 common targets seen in both the TNBC cells, i.e., TM9SF3, MEGF11, and GABRP. It has already been reported that MEGF11 and GABRP are required for TNBC cell proliferation [22, 23]. Thus, we focused on TM9SF3 and conducted a validation experiment using individual siRNAs against TM9SF3. We found that knockdown of TM9SF3 prevented the proliferation of MDA-MB-231 and MDA-MB-468 cells induced by FUCA2 (Figs. 5B and 5C). Furthermore, depletion of TM9SF3 diminished FUCA2-induced colony formation (Fig. 5D) and migration (Fig. 5E) in TNBC cells. Therefore, FUCA2-meidated TNBC cell aggressiveness shows a dependence on TM9SF3.

\section{Silencing of TM9SF3 impairs CAA-induced TNBC cell growth and invasion}

Next, we validated whether TM9SF3 is required for CAA-induced aggressive phenotype. To address this, we exposed TM9SF3-depleted TNBC cells to CAA conditioned media. Compared to control cells, TM9SF3- 
deficient MDA-MB-231 and MDA-MB-468 cells showed less profound growth (Fig. 6A), colony formation (Fig. 6B), and migration (Fig. 6C) upon stimulation with CAA conditioned media. These data suggest the importance of TM9SF3 in the crosstalk between CAA and TNBC progression.

\section{Tm9sf3 Overexpression Is A Poor Prognostic Factor For Tnbc}

Given the in vitro results indicating the involvement of TM9SF3 in TNBC aggressiveness, we then evaluated the clinical significance of TM9SF3 in this malignancy. Compared to adjacent nontumorous breast tissues, TNBC tissues had increased expression of TM9SF3, as determined by immunohistochemistry (Figs. 7A and 7B). Moreover, high TM9SF3 level was significantly associated with TNM stage and lymph node metastasis (Table 1). In addition, analysis of breast cancer RNA-seq data using Kaplan-Meier Plotter revealed that patients with high expression of TM9SF3 in breast cancer had significantly shorter overall survival than those with low expression of TM9SF3 ( $P=0.023$; Fig. 7C).

Table 1

Correlation of TM9SF3 expression with clinicopathologic characteristics of TNBC patients $(n=67)$

\begin{tabular}{|c|c|c|c|c|}
\hline \multirow[t]{2}{*}{ Variable } & \multirow[t]{2}{*}{$\mathbf{n}$} & \multicolumn{2}{|c|}{ TM9SF3 expression } & \multirow[t]{2}{*}{$P$} \\
\hline & & Low $(n=26)$ & High $(n=41)$ & \\
\hline Age (years) & & & & 0.8071 \\
\hline$<60$ & 27 & 10 & 17 & \\
\hline$\geq 60$ & 40 & 16 & 24 & \\
\hline TNM stage & & & & 0.0003 \\
\hline$I-I I$ & 33 & 20 & 13 & \\
\hline III-IV & 34 & 6 & 28 & \\
\hline Lymph node metastasis & & & & 0.0024 \\
\hline Negative & 26 & 16 & 10 & \\
\hline Positive & 41 & 10 & 31 & \\
\hline
\end{tabular}

\section{Discussion}

Our data show that FUCA2 expression is significantly elevated in CAAs relative to control adipocytes. Moreover, CAAs abundantly secrete FUCA2 protein to the extracellular environment, which raises the possibility that FUCA2 may mediate the crosstalk between CAAs and cancer cells. To test this hypothesis, we blocked FUCA2 activity using the neutralizing antibody. We found that blockade of FUCA2 activity 
dramatically suppresses the proliferation, colony formation, and migration of TNBC cells upon stimulation with CAA conditioned media. In contrast, exposure to recombinant FUCA2 protein augments the proliferative and migratory capacities of TNBC cells, supporting the hypothesis that FUCA2 plays an essential role in mediating CAA-induced aggressive phenotype of TNBC cells.

Multiple cell types in the tumor microenvironment are involved in tumor progression [24, 25]. For instance, cancer-associated fibroblasts recruit monocytes to enhance TNBC aggressiveness [25]. Takehara et al [26] reported that CAAs can produce SAA1 to enhance the migration/invasion capability and chemoresistance of pancreatic cancer cells. CAAs also confer more aggressive behaviors to breast cancer cells [8]. A previous study has suggested that CAAs can secret G-CSF to promote breast cancer malignant progression [27]. In the current study, we identify a novel secretory protein involved in CAAinduced aggressiveness of TNBC. Although FUCA2 has shown the diagnostic potential in ESCC and HCC $[10,11]$, its biological role in tumor progression, especially in TNBC is largely unknown. We provide direct evidence for the oncogenic activity of FUCA2 in TNBC. We demonstrated that TNBC cells exposed to FUCA2 exhibit increased growth and migratory abilities. In vivo studies confirmed that FUCA2 exposure reinforces tumorigenesis and lung metastasis of TNBC cells. Taken together, our results point toward the importance of FUCA2 in driving TNBC growth and metastasis.

Mechanistic investigation reveals that TM9SF3 is indispensable for FUCA2-elicited aggressive phenotype in TNBC. When TM9SF3 was silenced in TNBC cells, FUCA2-induced proliferation, colony formation, and migration were markedly impaired. Consistently, CAA-induced aggressive phenotype was also reversed by TM9SF3 knockdown. These results establish a requirement for TM9SF3 in the crosstalk between CAAs and TNBC cells. Although several other TM9SF family members such as TM9SF2 and TM9SF4 have been extensively studied in tumor progression $[15,16,28]$, the function of TM9SF3 is poorly determined. Our data show that TM9SF3 is significantly upregulated in TNBC tissues relative to adjacent breast tissues. Moreover, increased expression of TM9SF3 is associated with TNM stage, lymph node metastasis, and reduced overall survival. These results suggest that TM9SF3 plays an important role in TNBC. In agreement with this speculation, depletion of TM9SF3 counteracts CAA- and FUCA2-induced proliferation and migration of TNBC cells. However, knockdown of TM9SF3 has a little direct impact on the aggressive behaviors of TNBC cells. Therefore, TM9SF3 plays an essential role in the response of TNBC cells to tumor microenvironment.

On the basis of our results, we propose a model where CAAs release FUCA2 to enhance TNBC cell proliferation, colony formation, and migration through TM9SF3-dependent signaling (Fig. 7D). However, it remains to be determined how FUCA2 interacts with TM9SF3 in TNBC cells. In addition, the key signaling cascades downstream the interaction between FUCA2 and TM9SF3 need to be further clarified.

\section{Conclusions}

In conclusion, we identify FUCA2 as a CAA secretory protein, which can accelerate TNBC growth and metastasis through TM9SF3-dependent signaling. Clinically, TM9SF3 upregulation is associated with 
more aggressive behaviors of TNBC. Thus, TM9SF3 may serve as a promising target for blocking the crosstalk between TNBC cells and CAAs.

\section{Declarations}

\section{Ethics approval and consent to participate}

This study was approved by the Ethics Committee of First Affiliated Hospital of China Medical University (Shenyang, China). Written informed consent for research was obtained from each participant. All animal experiments were approved by the Animal Care Committee of China Medical University.

\section{Consent for publication}

Not applicable.

\section{Availability of data and materials}

Not applicable.

\section{Competing interests}

The authors declare that they have no competing interests.

\section{Funding}

This work was supported by the National Natural Science Foundation of China (81702593) and the Scientific Research Foundation of Educational Commission of Liaoning Province of China (LK201629).

\section{Authors' contributions}

PX and FJ designed the experiments, processed the experimental data, and drafted the manuscript. HD, $\mathrm{QL}, \mathrm{TZ}, \mathrm{FY}, \mathrm{YX}, \mathrm{BC}$, and YW conducted the experiments and analyzed the data. All authors read and approved the final version of the manuscript.

\section{Acknowledgements}

None.

\section{References}

1. Chaudhary LN. Early stage triple negative breast cancer: Management and future directions. Semin Oncol. 2020;S0093-7754(20):30045-2.

2. Savas P, Loi S. Expanding the Role for Immunotherapy in Triple-Negative Breast Cancer. Cancer Cell. 2020;37:623-4. 
3. Keren L, Bosse M, Marquez D, Angoshtari R, Jain S, Varma S, Yang SR, Kurian A, Van Valen D, West R, Bendall SC, Angelo M. A Structured Tumor-Immune Microenvironment in Triple Negative Breast Cancer Revealed by Multiplexed Ion Beam Imaging. Cell. 2018;174:1373-87.

4. Gruosso T, Gigoux M, Manem VSK, Bertos N, Zuo D, Perlitch I, Saleh SMI, Zhao H, Souleimanova M, Johnson RM, Monette A, Ramos VM, Hallett MT, Stagg J, Lapointe R, Omeroglu A, Meterissian S, Buisseret L, Van den Eynden G, Salgado R, Guiot MC, Haibe-Kains B, Park M. Spatially distinct tumor immune microenvironments stratify triple-negative breast cancers. J Clin Invest. 2019;129:1785800 .

5. Zewdu A, Casadei L, Pollock RE, Braggio D. Adipose Tumor Microenvironment. Adv Exp Med Biol. 2020;1226:73-86.

6. Wu Q, Li B, Li Z, Li J, Sun S, Sun S. Cancer-associated adipocytes: key players in breast cancer progression. J Hematol Oncol. 2019;12:95.

7. Sabol RA, Bowles AC, C?té A, Wise R, O'Donnell B, Matossian MD, Hossain FM, Burks HE, Del Valle L, Miele L, Collins-Burow BM, Burow ME, Bunnell BA. Leptin produced by obesity-altered adipose stem cells promotes metastasis but not tumorigenesis of triple-negative breast cancer in orthotopic xenograft and patient-derived xenograft models. Breast Cancer Res. 2019;21:67.

8. Dirat B, Bochet L, Dabek M, Daviaud D, Dauvillier S, Majed B, Wang YY, Meulle A, Salles B, Le Gonidec S, Garrido I, Escourrou G, Valet P, Muller C. Cancer-associated adipocytes exhibit an activated phenotype and contribute to breast cancer invasion. Cancer Res. 2011;71:2455-65.

9. Grootaert H, Van Landuyt L, Hulpiau P, Callewaert N. Functional exploration of the GH29 fucosidase family. Glycobiology. 2020;30:735-45.

10. Yu X, Zhang R, Yang T, Zhang M, Xi K, Lin Y, Wen Y, Wang G, Huang Z, Zhang X, Zhang L. Alpha-Ifucosidase: a novel serum biomarker to predict prognosis in early stage esophageal squamous cell carcinoma. J Thorac Dis. 2019;11:3980-90.

11. Waidely E, Al-Youbi AO, Bashammakh AS, El-Shahawi MS, Leblanc RM. Alpha-I-Fucosidase Immunoassay for Early Detection of Hepatocellular Carcinoma. Anal Chem. 2017;89:9459-66.

12. Liu TW, Ho CW, Huang HH, Chang SM, Popat SD, Wang YT, Wu MS, Chen YJ, Lin CH. Role for alpha-Lfucosidase in the control of Helicobacter pylori-infected gastric cancer cells. Proc Natl Acad Sci U S A. 2009;106:14581-6.

13. Perrin J, Le Coadic M, Vernay A, Dias M, Gopaldass N, Ouertatani-Sakouhi H, Cosson P. TM9 family proteins control surface targeting of glycine-rich transmembrane domains. J Cell Sci. 2015;128:2269-77.

14. Lozupone F, Borghi M, Marzoli F, Azzarito T, Matarrese P, lessi E, Venturi G, Meschini S, Canitano A, Bona R, Cara A, Fais S. TM9SF4 is a novel V-ATPase-interacting protein that modulates tumor $\mathrm{pH}$ alterations associated with drug resistance and invasiveness of colon cancer cells. Oncogene. 2015;34:5163-74.

15. Zhu Y, Xie M, Meng Z, Leung LK, Chan FL, Hu X, Chi K, Liu C, Yao X. Knockdown of TM9SF4 boosts ER stress to trigger cell death of chemoresistant breast cancer cells. Oncogene. 2019;38:5778-91. 
16. Li Q, Lei C, Lu C, Wang J, Gao M, Gao W. LINC01232 exerts oncogenic activities in pancreatic adenocarcinoma via regulation of TM9SF2. Cell Death Dis. 2019;10:698.

17. Shen L, Du X, Ma H, Mei S. miR-1193 Suppresses the Proliferation and Invasion of Human T-Cell Leukemia Cells Through Directly Targeting the Transmembrane 9 Superfamily 3 (TM9SF3). Oncol Res. 2017;25:1643-51.

18. Nieman KM, Kenny HA, Penicka CV, Ladanyi A, Buell-Gutbrod R, Zillhardt MR, Romero IL, Carey MS, Mills GB, Hotamisligil GS, Yamada SD, Peter ME, Gwin K, Lengyel E. Adipocytes promote ovarian cancer metastasis and provide energy for rapid tumor growth. Nat Med. 2011;17:1498-503.

19. Wang H, Rangaswamy S, Kodavati M, Mitra J, Guo W, Guerrero EN, Van Den Bosch L, Hegde ML. RT(2) PCR array screening reveals distinct perturbations in DNA damage response signaling in FUSassociated motor neuron disease. Mol Brain. 2019;12:103.

20. Mahmood SF, Gruel N, Chapeaublanc E, Lescure A, Jones T, Reyal F, Vincent-Salomon A, Raynal V, Pierron G, Perez F, Camonis J, Del Nery E, Delattre O, Radvanyi F, Bernard-Pierrot I. A siRNA screen identifies RAD21, EIF3H, CHRAC1 and TANC2 as driver genes within the 8q23, 8q24.3 and 17q23 amplicons in breast cancer with effects on cell growth, survival and transformation. Carcinogenesis. 2014;35:670-82.

21. Deshmukh AS, Peijs L, Beaudry JL, Jespersen NZ, Nielsen CH, Ma T, Brunner AD, Larsen TJ, BayarriOlmos R, Prabhakar BS, Helgstrand C, Severinsen MCK, Holst B, Kjaer A, Tang-Christensen M, Sanfridson A, Garred P, Privé GG, Pedersen BK, Gerhart-Hines Z, Nielsen S, Drucker DJ, Mann M, Scheele C. Proteomics-Based Comparative Mapping of the Secretomes of Human Brown and White Adipocytes Reveals EPDR1 as a Novel Batokine. Cell Metab. 2019;30:963-75.

22. Chiu JH, Tseng LM, Huang TT, Liu CY, Wang JY, Huang CP, Tsai YF, Hsu CY. MEGF11 is related to tumour recurrence in triple negative breast cancer via chemokine upregulation. Sci Rep. 2020;10:8060.

23. Wali VB, Patwardhan GA, Pelekanou V, Karn T, Cao J, Ocana A, Yan Q, Nelson B, Hatzis C, Pusztai L. Identification and Validation of a Novel Biologics Target in Triple Negative Breast Cancer. Sci Rep. 2019;9:14934.

24. Hollmén M, Karaman S, Schwager S, Lisibach A, Christiansen AJ, Maksimow M, Varga Z, Jalkanen S, Detmar M. G-CSF regulates macrophage phenotype and associates with poor overall survival in human triple-negative breast cancer. Oncoimmunology. 2015;5:e1115177.

25. Allaoui R, Bergenfelz C, Mohlin S, Hagerling C, Salari K, Werb Z, Anderson RL, Ethier SP, Jirstr?m K, PåhIman S, Bexell D, Tahin B, Johansson ME, Larsson C, Leandersson K. Cancer-associated fibroblast-secreted CXCL16 attracts monocytes to promote stroma activation in triple-negative breast cancers. Nat Commun. 2016;7:13050.

26. Takehara M, Sato Y, Kimura T, Noda K, Miyamoto H, Fujino Y, Miyoshi J, Nakamura F, Wada H, Bando Y, Ikemoto T, Shimada M, Muguruma N, Takayama T. Cancer-associated adipocytes promote pancreatic cancer progression through SAA1 expression. Cancer Sci. 2020;111:2883-94. 
27. Liu L, Wu Y, Zhang C, Zhou C, Li Y, Zeng Y, Zhang C, Li R, Luo D, Wang L, Zhang L, Tu S, Deng H, Luo S, Chen YG, Xiong X, Yan X. Cancer-associated adipocyte-derived G-CSF promotes breast cancer malignancy via Stat3 signaling. J Mol Cell Biol. 2020;12:723-37.

28. Li Q, Lei C, Lu C, Wang J, Gao M, Gao W. LINC01232 exerts oncogenic activities in pancreatic adenocarcinoma via regulation of TM9SF2. Cell Death Dis. 2019;10:698.

Figures


\section{Control CAA}

FUCA2

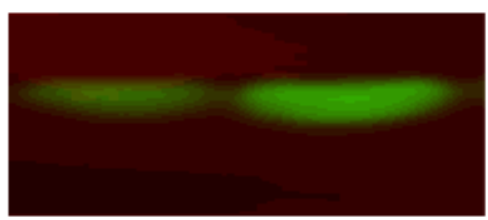

GAPDH



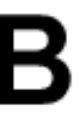

Control

CAA

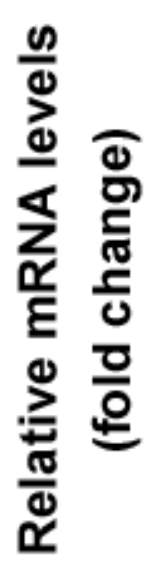

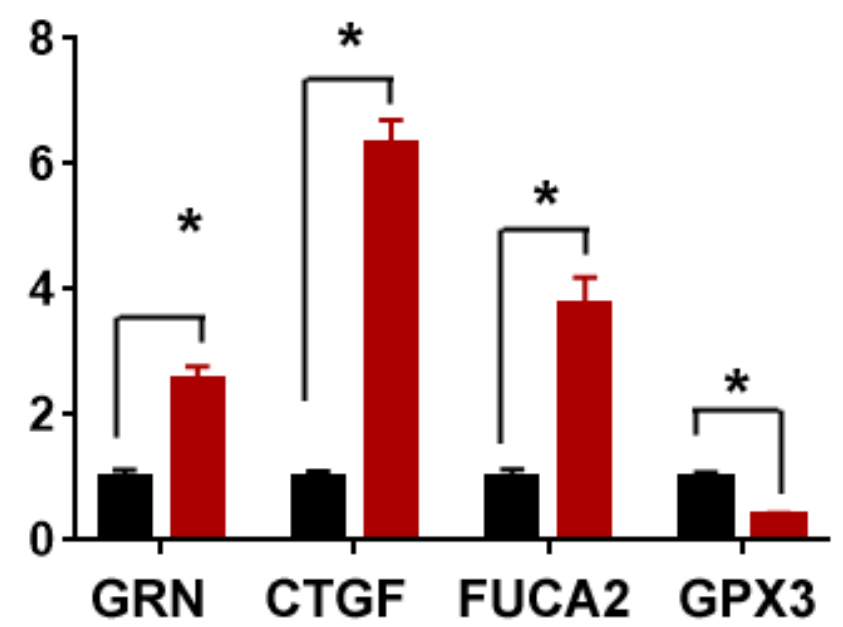



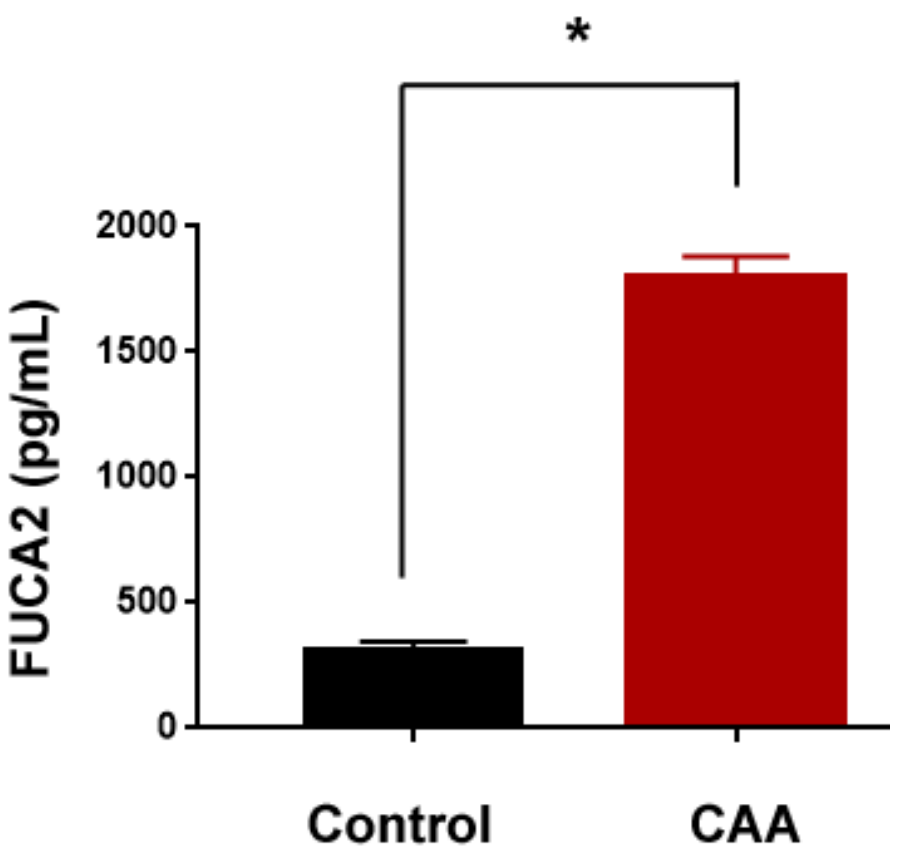

Figure 1 
Increased production of FUCA2 by CAAs isolated from TNBC patients. (A) Flow chart of detection of differentially expressed genes in CAAs and control adipocytes. (B) Quantification of GRN, CTGF, FUCA2, and GPX3 mRNA levels in CAAs and control adipocytes. (C) Western blot analysis of FUCA2 protein in CAAs and control adipocytes. (D) ELISA analysis of FUCA2 concentrations in conditioned media of CAAs and control adipocytes. * $P<0.05$.
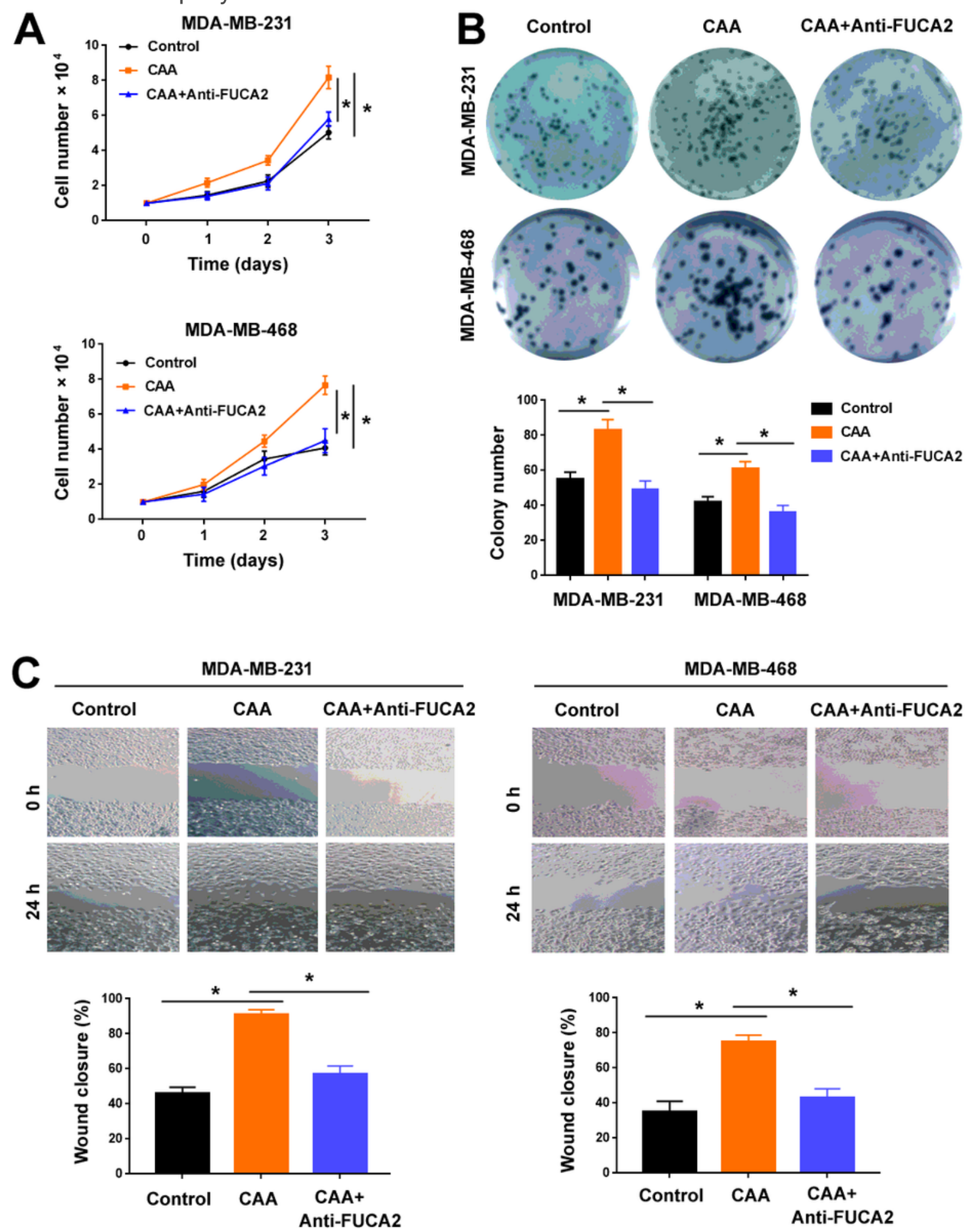

Figure 2 
Blocking FUCA2 attenuates CAA-induced aggressiveness in TNBC cells. (A) CAA conditioned media promoted the proliferation of TNBC cells, and blocking FUCA2 attenuated CAA-induced proliferation. (B) Colony formation assay performed in TNBC cells treated as in (A). Bottom, quantitative results from three independent experiments. (C) Cell migration was determined by in vitro wound-healing assay. Bottom, quantitative results from three independent experiments. ${ }^{*} \mathrm{P}<0.05$.
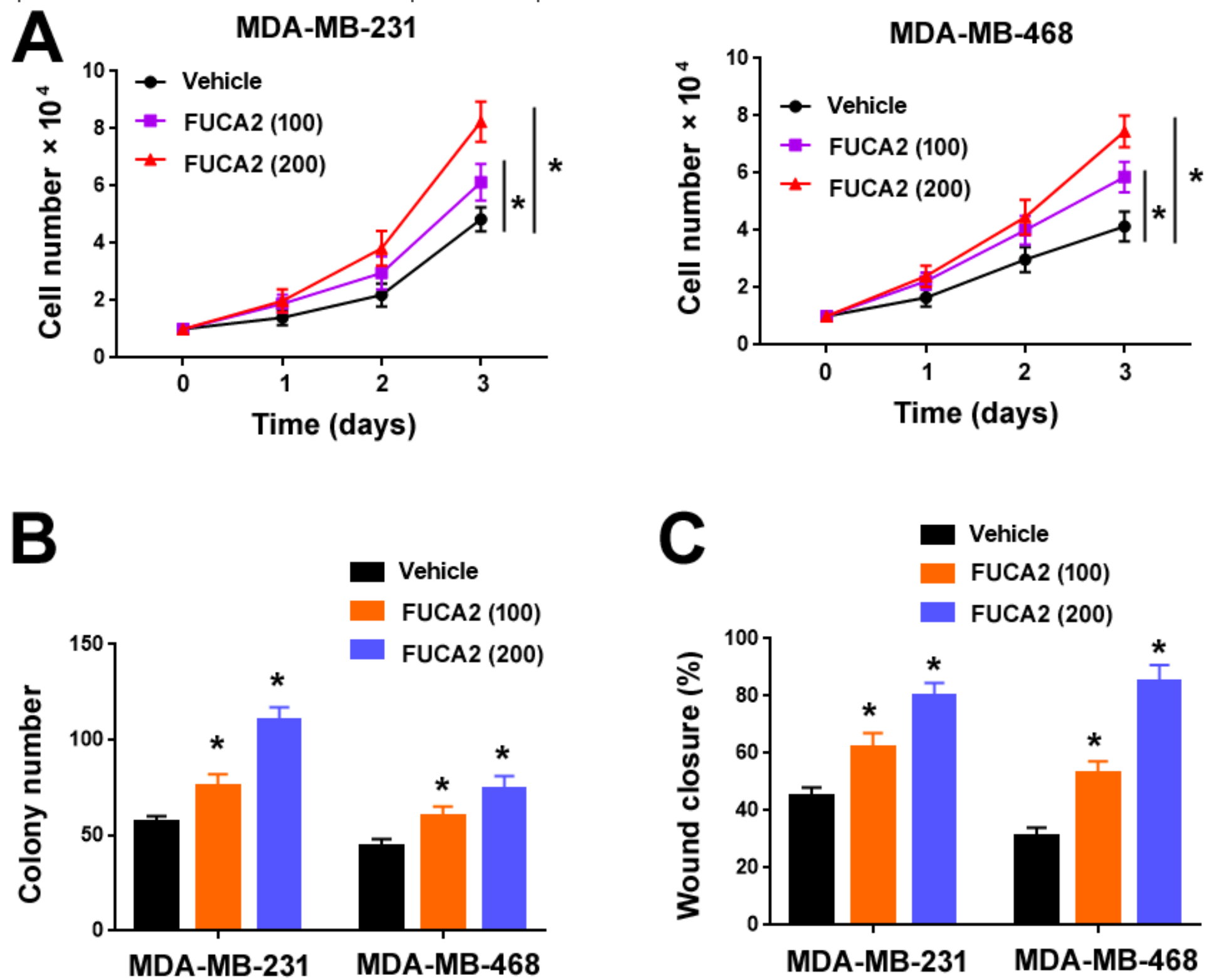

Figure 3

Exogenous FUCA2 drives TNBC cell growth and migration. (A) FUCA2 treatment promoted the proliferation of TNBC cells. (B) FUCA2 treatment enhanced the colony formation ability of TNBC cells. (C) The invasive ability of TNBC cells were increased in the presence of FUCA2. ${ }^{*} \mathrm{P}<0.05$ vs. vehicle. FUCA2 (100): 100 ng/mL FUCA2; FUCA2 (200): 200 ng/mL FUCA2. 


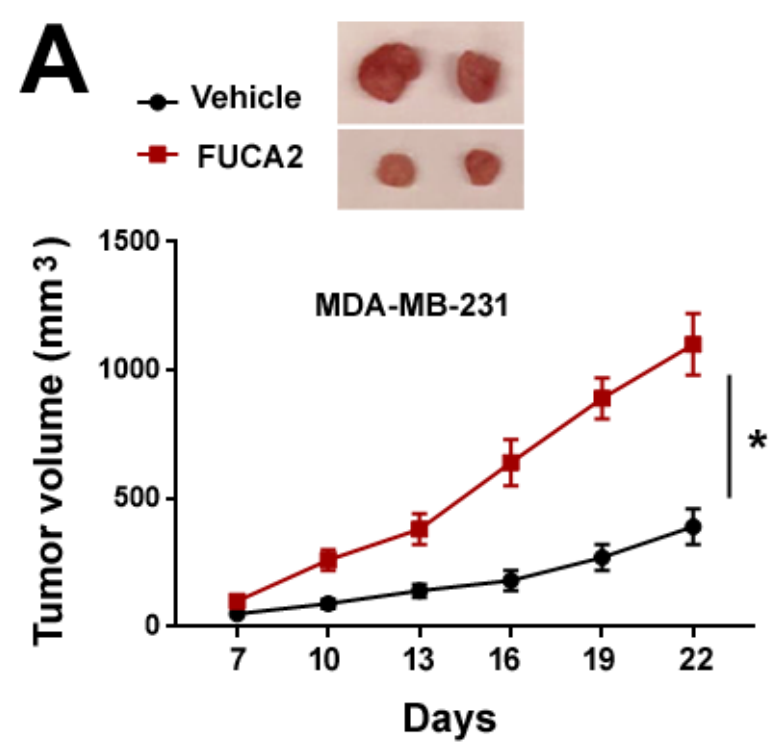

B
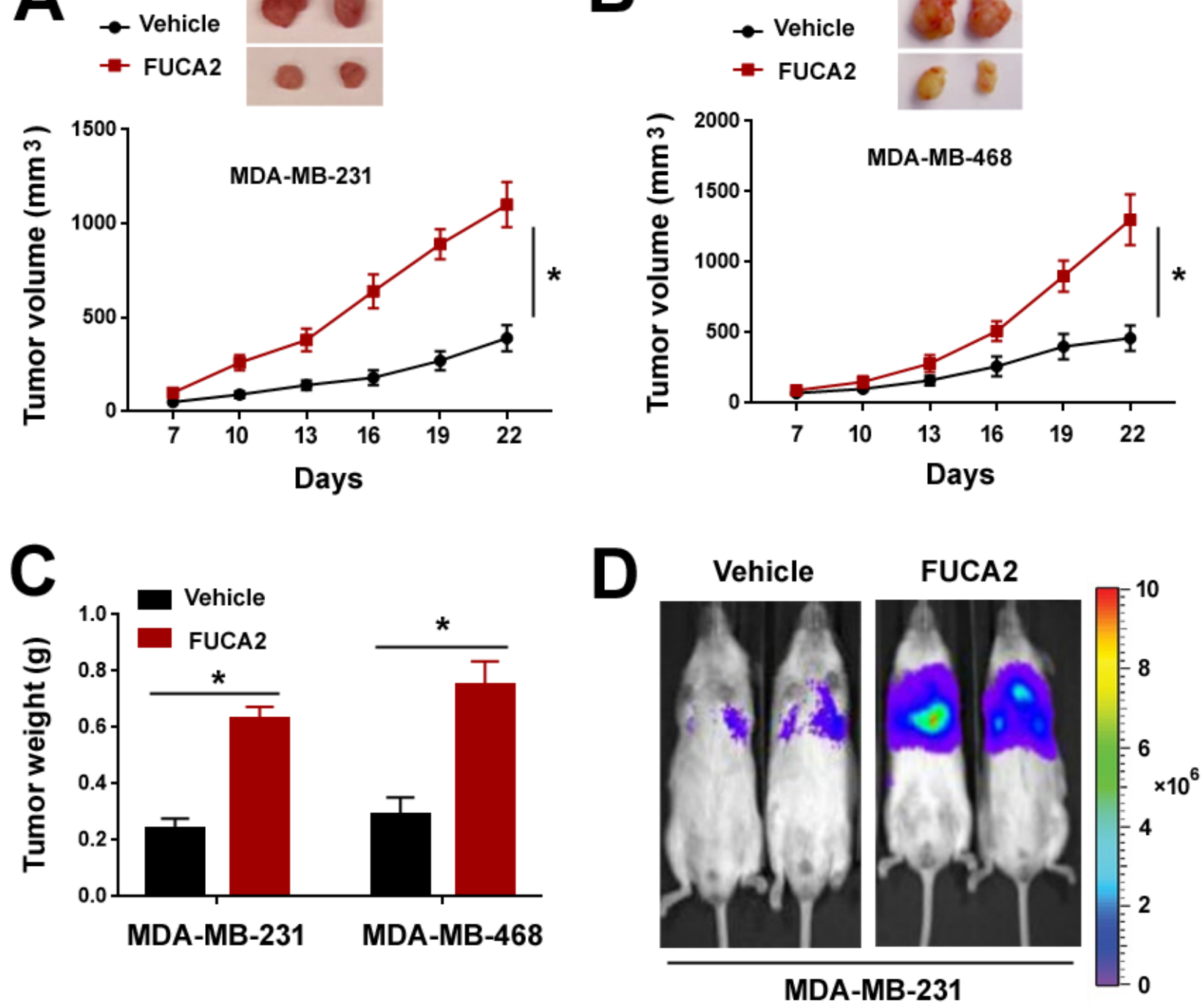

$E$



Figure 4

FUCA2 exposure promotes tumorigenesis and metastasis of TNBC cells in vivo. (A-C) Tumorigenic studies. TNBC cells were exposed to $200 \mathrm{ng} / \mathrm{mL}$ FUCA2 protein for 1 week and injected subcutaneously into nude mice. Tumor growth curves were plotted in $(A)$ and $(B) ; n=4$. Inserts are representative tumors from 2 mice of each group. (C) Measurement of the weight of xenograft tumors $(n=4)$. (D,E) Assessment 
of tumor metastasis. (D) Two representative mice from each group. (E) Measurement of bioluminescence levels in the lung $(n=4)$. ${ }^{*}<<0.05$.

A
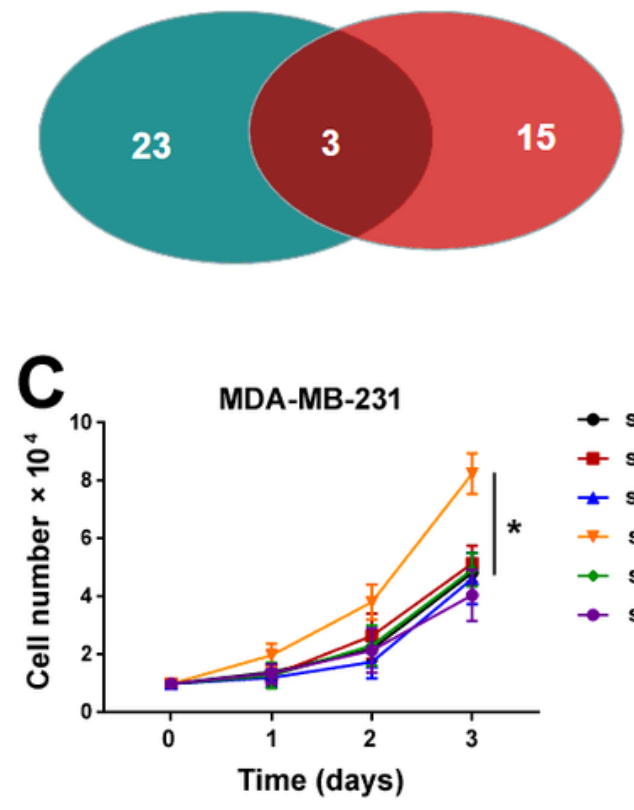

D

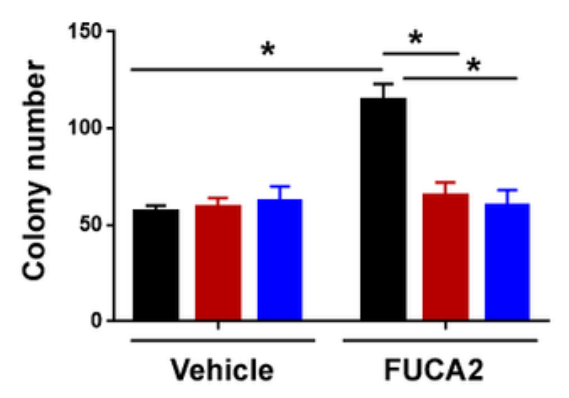

E



B

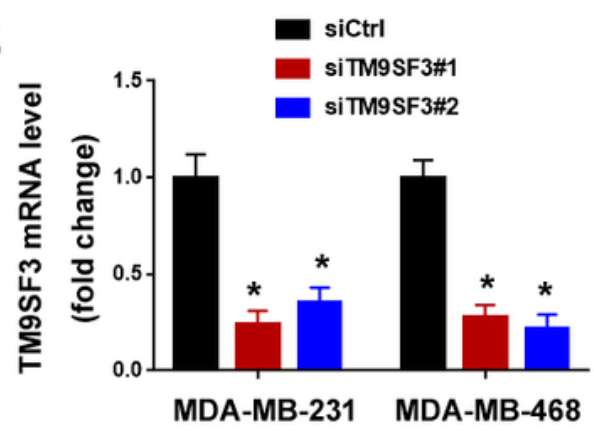

MDA-MB-468

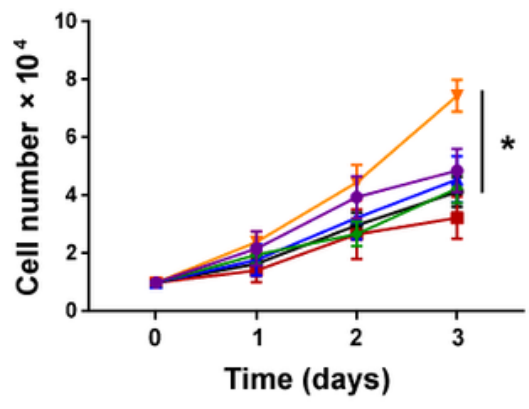

MDA-MB-468

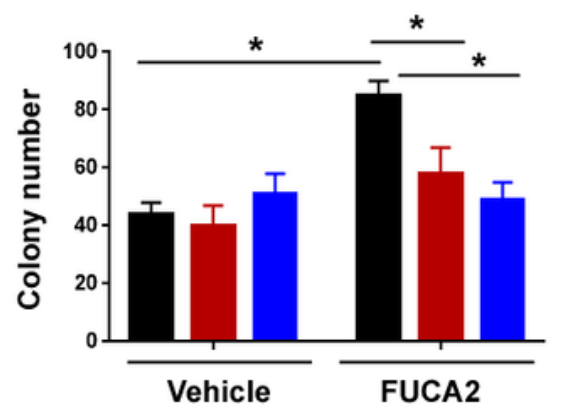

MDA-MB-468

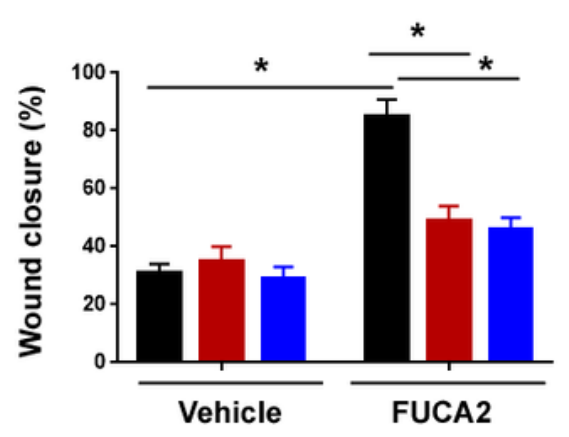

Figure 5

FUCA2-mediated TNBC aggressiveness depends on TM9SF3 expression. (A) High-throughput siRNA screening studies. Venn diagram showing the identification of 3 common genes whose knockdown blocked FUCA2-induced TNBC cell proliferation. (B) Transfection of TM9SF3-targeting siRNAs 
(siTM9SF3\#1,2) led to downregulation of TM9SF3 relative to control siRNA (siCtrl). ${ }^{*} \mathrm{P}<0.05$ vs. siCtrl. (C) Measurement of proliferation in cells transfected with indicated siRNAs and treated with or without FUCA2. (D) Colony formation assay in the cells with indicated treatments. (E) Migration assay in the cells with indicated treatments. ${ }^{*} \mathrm{P}<0.05$.
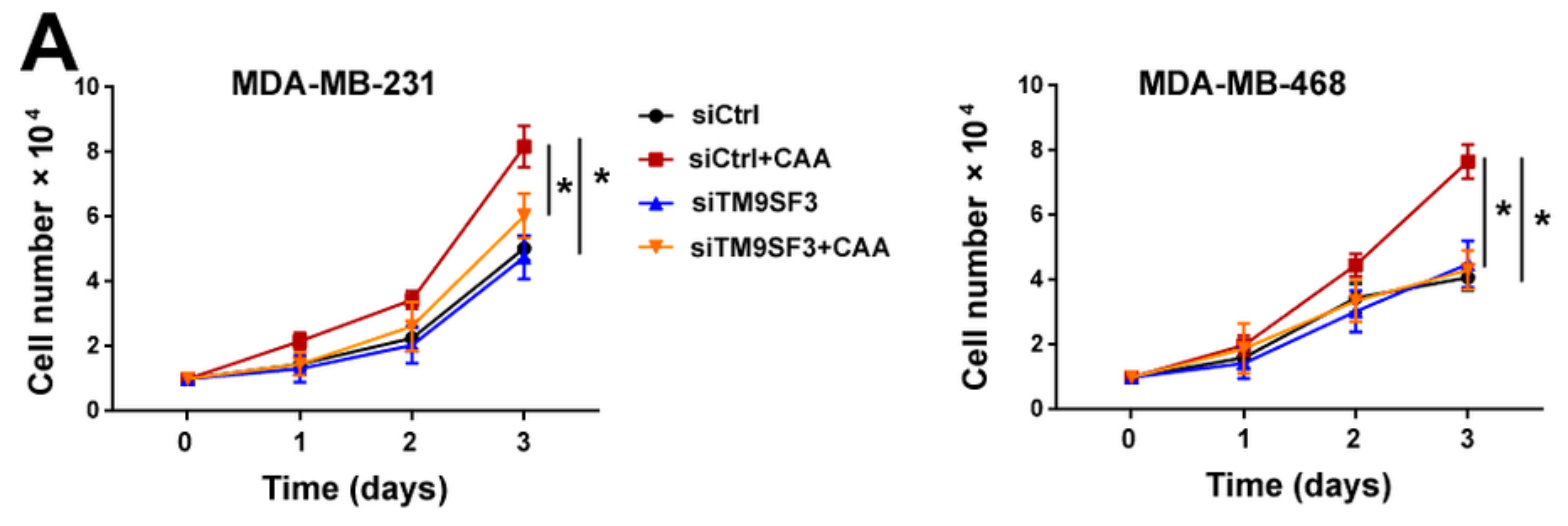

B

MDA-MB-231

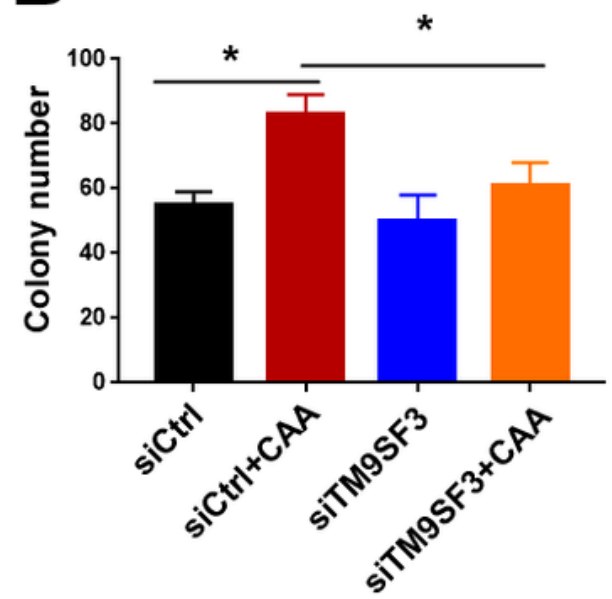

C

MDA-MB-231

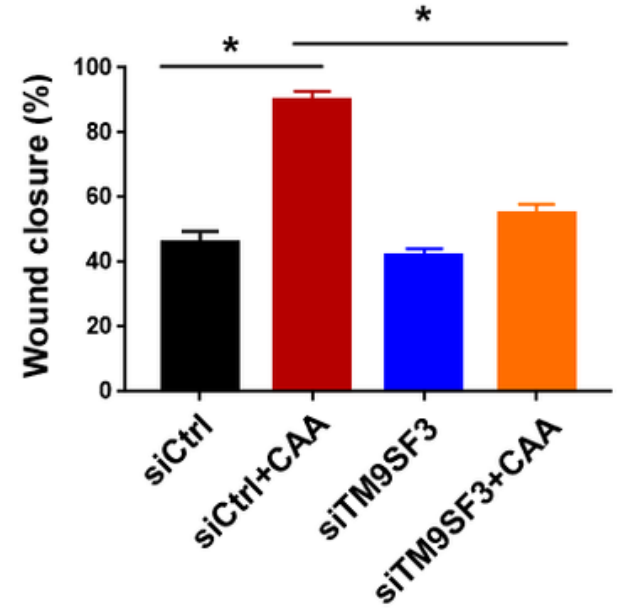

MDA-MB-468



MDA-MB-468

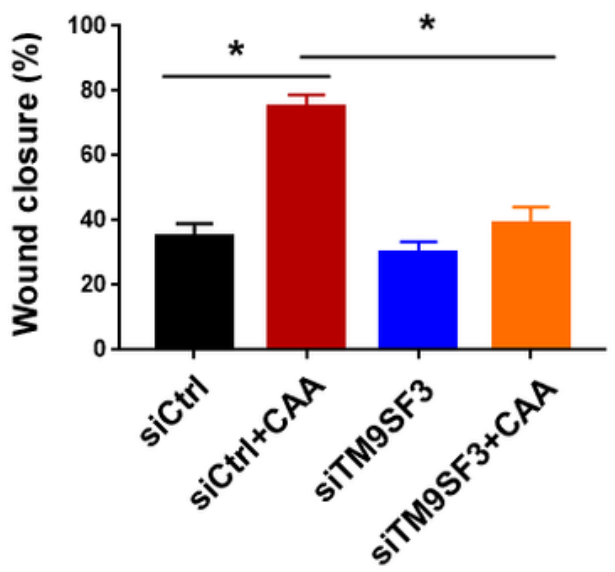

Figure 6 
Silencing of TM9SF3 impairs CAA-induced TNBC cell growth and invasion. TNBC cells were transfected with siTM9SF3 or siCtrl and then exposed to CAA conditioned media or PBS (vehicle). Measurement of (A) cell proliferation, $(B)$ colony formation, and (C) migration. * $\mathrm{P}<0.05$.

A
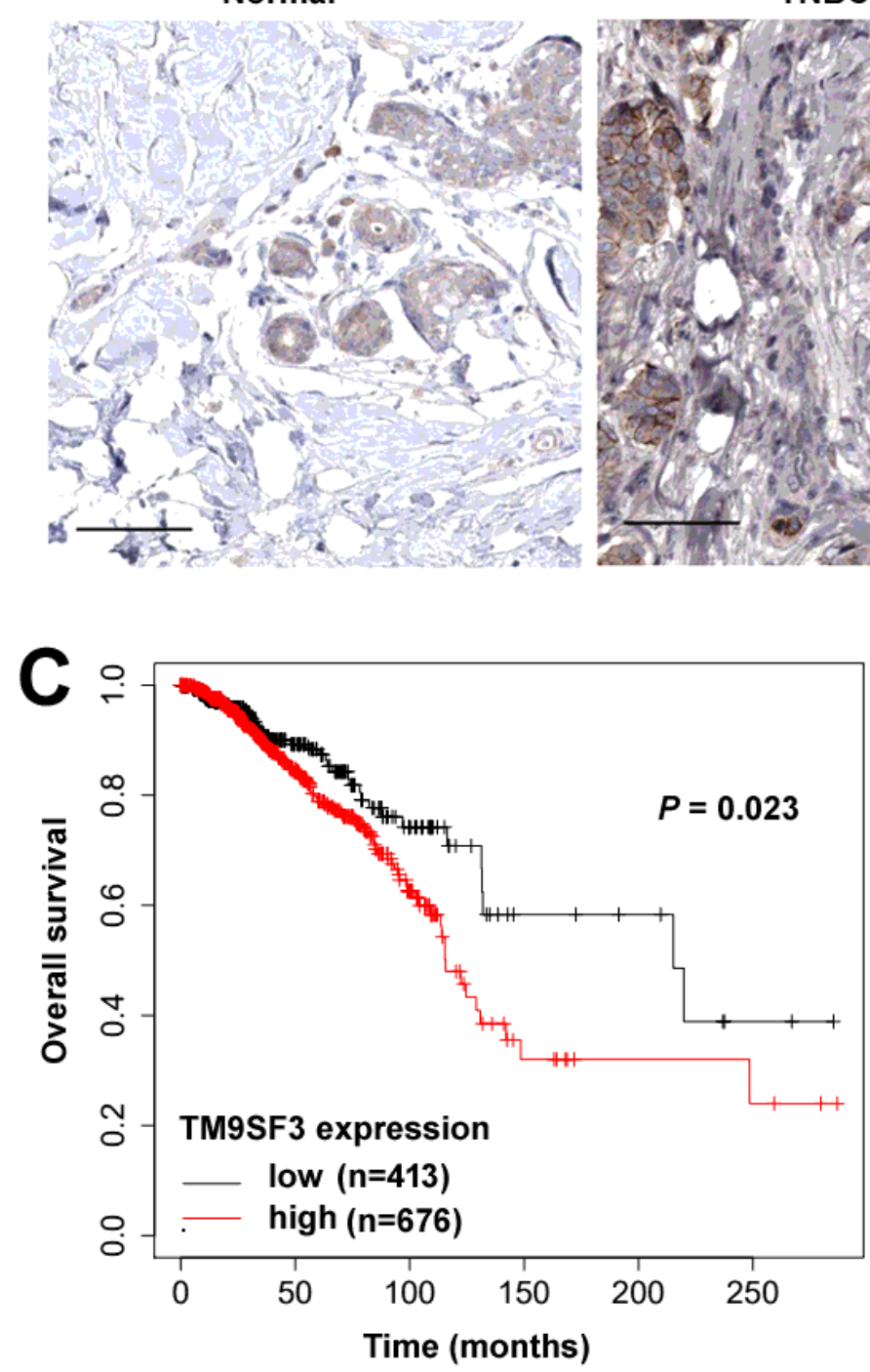

TNBC

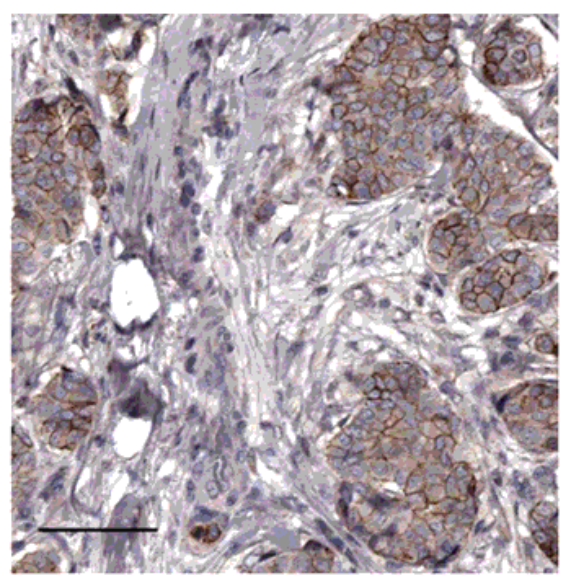

D

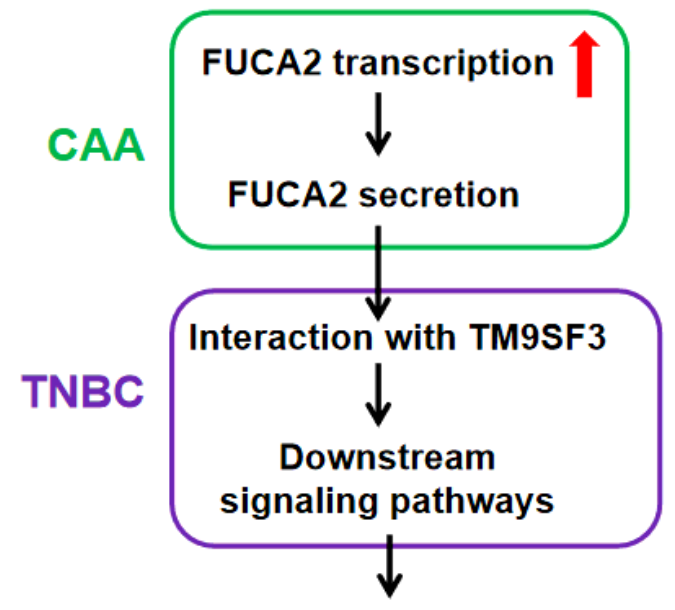

TNBC growth and metastasis

\section{Figure 7}

TM9SF3 overexpression is a poor prognostic factor for TNBC. $(A, B)$ Immunohistochemical analysis of TM9SF3 in TNBC and adjacent nontumorous breast tissues. Scale bar $=100 \mu \mathrm{m}$. (B) Immunohistochemical scores were determined $(n=67)$. (C) Survival curves were plotted for breast cancer patients with different expression levels of TM9SF3. Data was analyzed using Kaplan-Meier Plotter. (D) Schematic model showing that CAAs secretes FUCA2 to enhance TNBC growth and metastasis through interaction with TM9SF3. 